\title{
Natura@economía
}

ISSN 2226-9479 (Versión electrónica) Website: http://revistas.lamolina.edu.pe/index.php/neu

ARTÍCULO ORIGINAL

\section{Implementación de la contabilidad gerencial para la efectiva toma de decisiones en una microempresa gráfica de Lima Metropolitana}

Implementation of managerial accounting for effective decision making in a graphic microenterprise of Metropolitan Lima

\section{Luis Eduardo Jr. Correa Chamorro ${ }^{1 *}$; Pedro Ricardo Quiroz Quezada ${ }^{2}$; José Luis Allcca Alzamora ${ }^{3}$}

\footnotetext{
${ }^{1}$ Universidad Nacional Agraria la Molina, Lima, Perú. ID de ORCID: 0000-0001-7930-7969, lucoch74@ gmail.com.

${ }^{2}$ Universidad Nacional Agraria la Molina, Lima, Perú. ID de ORCID: 0000-0003-2981-8087, pquiroz@ lamolina.edu.pe.

${ }^{3}$ Universidad Nacional Agraria la Molina, Lima, Perú. ID de ORCID: 0000-0001-9252-7966, jalca@lamolina.edu.pe.
}

Recepción: 22/3/2021; Aceptación: 16/5/2021

\section{Resumen}

La investigación tuvo como objetivo demostrar que la implementación de la contabilidad gerencial efectiviza la toma decisiones permitiendo tener mayores utilidades. La investigación es de tipo Aplicada, con enfoque cuantitativo, de alcance Descriptivo - Explicativo, bajo método Inductivo - Deductivo y de diseño Experimental. La población fue de nueve personas, no se aplicó muestreo probabilístico. Las técnicas empleadas fueron un cuestionario y análisis documental. Los instrumentos usados fueron la encuesta y reportes de gestión. Los resultados se procesaron con Microsoft Excel $365^{\circ}$ y SPSS v.26. La prueba estadística utilizada para medir la influencia de las variables fue el T-Student. La contabilidad gerencial contribuye directa y efectivamente, en la toma de decisiones empresariales de la microempresa gráfica de Lima Metropolitana.

Palabras clave: Contabilidad gerencial; toma de decisiones; microempresa gráfica; cuadro de mando integral; ratios financieros.

Forma de referenciar el artículo: Correa, L., Quiroz, P. y Allcca, J. (2021). Implementación de la contabilidad gerencial para la efectiva toma de decisiones en una microempresa gráfica de Lima Metropolitana, Natura@ economía, 6(1), 43-59. http://dx.doi.org/10.21704/ne.v6i1.1730

DOI: http://dx.doi.org/10.21704/ne.v6i1.1730

* Autor de correspondencia: Correa, L. Email: lucoch74@gmail.com

CLos autores. Publicado por la Universidad Nacional Agraria La Molina. 


\begin{abstract}
The objective of the research was demonstrate that the implementation of managerial accounting makes decision-making effective, allowing higher profits. The research is Applied, with a quantitative approach, descriptive - explanatory scope, under Inductive - Deductive method and Experimental design. The population consisted of nine people, probability sampling was not applied. The techniques used were the questionnaire and documentary analysis. The instruments used were the survey and management reports. The results were processed with Microsoft Excel $365^{\circ}$ and SPSS v.26. The statistical test used to measure the influence of the variables was the T-student. Managerial accounting contributes directly and effectively to business decisionmaking of the graphic microenterprise of Metropolitan Lima.
\end{abstract}

Keywords: Managerial Accounting; business decision making; graphic microenterprise; balanced scorecard; financial ratios.

\section{Introducción}

El panorama actual de las microempresas gráficas, muestran la necesidad de aplicar los métodos y técnicas de la contabilidad gerencial que, unido al grado de autonomía de las organizaciones privadas, proporcionará al directivo parámetros de control que permitirá facilitar la toma de decisiones y tener una gestión eficiente.

La limitación fundamental que presenta las microempresas, en general, es no evaluar con antelación, las decisiones afectaran la situación económica, patrimonial, talento humano y producción.

Las microempresas gráficas, sobre todo en el Perú, presentan grandes problemas en su gestión administrativa, producción, alta rotación del talento humano e inestable control contable al no considerar los riesgos de inversión, para crecer raudamente, lo cual hace que el negocio indirectamente se limite y tenga utilidades volubles, debido a decisiones improvisadas; causando el quiebre de las mismas en un plazo estimado de tres (03) años. La Sociedad de Comercio Exterior del Perú (2020), señala que el desarrollo económico del Perú está impulsado por las MYPES, quienes, durante el año 2019, conformaron el $95 \%$ del total de empresas, empleando el $47,7 \%$ de la Población Económicamente Activa y obteniendo una participación del 19,3\% del PBI nacional, motivo por el cual, son consideradas el "motor que dinamiza la productividad del país" e incrementan su capacidad de empleabilidad desde el sector privado.

Asimismo, según la Superintendencia Nacional de Administración Tributaria y Aduanas (2019), en concordancia con la Ley $\mathrm{N}^{\circ} 28015$ - Ley de Promoción y Formalización de la Micro y Pequeña Empresa en el Perú, describe que una microempresa se identifica por contar como máximo diez (10) trabajadores y un monto máximo de venta anual de 150 Unidades Impositivas Tributarias (UIT), del cual el valor de una (01) UIT en el año 2019 fue de S/ 4200,00. Por ello, el ejecutivo se convierte en un decididor sobre aspectos tales como: dónde obtener los recursos, en qué invertir, cuáles son los beneficios o utilidades, cuándo y cómo se le debe pagar a las fuentes de financiamiento y clientes internos. Entonces, se debe usar la contabilidad gerencial, empleando diversas técnicas y herramientas financieras, tales como: Ratios Financieros, Estado Situación Financiera, Estado de Resultados, Estado de Flujo de Caja, Ratios Financieros, Indicadores de Rentabilidad, Punto de Equilibrio, Grado de Apalancamiento, Gestión del Riesgo, entre otros, que son útiles para una efectiva toma de decisiones (Flores, 2019).

El objetivo de la investigación es demostrar que la implementación de la contabilidad 
gerencial permite tomar decisiones efectivas en una pequeña empresa grafica en Lima Metropolitana, el cual constituye una recopilación de información y esquematización de una solución con el empleo de la Contabilidad Gerencial, ajustada a la realidad de la microempresa gráfica; en base al Diagnóstico Situacional de la empresa, formulación y estructuración de las Herramientas Financieras y, posterior control y evaluación con el Cuadro de Mando Integral, quienes influyen directamente en la Toma de Decisiones de la organización. Para ello, se realizó un tratamiento metodológico para la mejor comprensión de cualquier persona interesada en la temática.

\section{Materiales y métodos}

\section{Lugar de la investigación}

El estudio se realizó en la microempresa gráfica, Copy Data E.I.R.L., con RUC $\mathrm{N}^{\circ}$ 20521018322, con más de diez (10) años de experiencia en el rubro, localizada en la Av. Benavides $\mathrm{N}^{\circ} 533$ Int. 62A - Urbanización Las Gardenias, distrito de Santiago de Surco, Lima Metropolitana, departamento de Lima, Perú.

Tipo, enfoque, alcance, método y diseño de la investigación

Según Hernandez-Sampieri y Mendoza (2018), la investigación es de tipo Aplicada, por involucrar a la contabilidad gerencial como solución metodológica teniendo como consecuencia una efectiva toma de decisiones; con enfoque Cuantitativo, por utilizar datos numéricos extraídos de los reportes de gestión y las encuestas; de alcance Descriptivo - Explicativo, por detallar, explicar y calcular con precisión la influencia de las dimensiones con la variable en estudio; bajo método Inductivo - Deductivo; y, de diseño Experimental, porque se manipuló el método de solución y la variable con sus dimensiones de la investigación, con la finalidad de tener datos fidedignos sobre la influencia de la contabilidad gerencial en los distintos tipos de toma de decisiones.

\section{Población y muestra}

Estaba conformada por toda la población trabajadora en la parte administrativa y operativa son de nueve (09) personas, tomando la alternativa de realizar un censo, para tener un análisis certero de los resultados de la investigación, sin margen de error. No se realizó ningún tipo de muestreo probabilístico.

\section{Variable y dimensiones de la investigación}

Tabla 1. Variable y dimensiones.

\begin{tabular}{cc}
\hline Variable & Dimensiones \\
Variable Única & $\mathbf{Y}_{\mathbf{1}}$. Decisión de Financiamiento \\
\cline { 2 - 2 } Y. & $\mathbf{Y}_{\mathbf{2}}$. Decisión sobre Inversión \\
\cline { 2 - 2 } Toma de decisiones & $\mathbf{Y}_{\mathbf{3}}$. Decisión sobre Riesgo \\
\cline { 2 - 2 } & $\mathbf{Y}_{\mathbf{4}}$. Decisión sobre Rentabilidad \\
\hline
\end{tabular}




\section{Solución metodológica}

Se empleó la Contabilidad Gerencial, como medio para tomar decisiones con información fidedigna y cuantificada, que brindan las herramientas financieras, tales como: Estado de Situación Financiera, Estado de Resultados y Ratios Financieros, el cual brinda información financiera; así como, el uso de la Matriz FODA (Fortalezas, Oportunidades, Debilidades y Amenazas) y el Cuadro de Mando Integral. Para ello, se brindó capacitaciones de 04 sesiones sobre el uso de estas herramientas a todo el personal.

\section{Técnicas de recolección y análisis de datos}

Las técnicas que se usaron fue el cuestionario y análisis documental. En este proceso se empleó dos tipos deinstrumentos, clasificándolos como metodológicos y teóricos; respecto al primer tipo, involucra la aplicación de una encuesta pre y post implementación de la contabilidad gerencial, compuesto por 16 preguntas bajo escala de respuestas Likert (1. Totalmente en Desacuerdo, 2. En Desacuerdo, 3. Ni de Acuerdo ni en Desacuerdo, 4. De Acuerdo y 5. Totalmente de Acuerdo), que aborda 4 ítem por dimensiones de la única variable; se determinó su validez bajo la valoración de juicio de expertos ante la formulación de las preguntas, que fueron acorde a la realidad de la microempresa y las teorías científicas acerca de la variable en mención; la fiabilidad del test se realizó mediante el análisis de consistencia interna, con la aplicación de la prueba en los 9 integrantes de la microempresa Copy Data E.I.R.L, obteniendo un coeficiente Alfa de Cronbach de 0,948, el cual implica un aceptable nivel de fiabilidad (Tabla 2). Sin embargo, respecto al segundo tipo, es pertinente a la demostración y efectos de la aplicación de la contabilidad gerencial, el cual abarca el análisis documental y estructuración de las Herramientas Financieras, Cuadro de Mando Integral y Análisis FODA.

Tabla 2. Aplicación del Pre y Post test de la Contabilidad Gerencial en cada dimensión y variable toma de decisiones.

\begin{tabular}{lccc}
\hline \multicolumn{1}{c}{ Toma de decisiones } & N $^{\mathrm{o}}$ Ítems & Pre-test & Post-test \\
\hline Decisión de Financiamiento & 4 & 0,812 & 0,806 \\
Decisión sobre Inversión & 4 & 0,808 & 0,826 \\
Decisión sobre Riesgo & 4 & 0,837 & 0,859 \\
Decisión sobre Rentabilidad & 4 & 0,809 & 0,818 \\
\multicolumn{1}{c}{ Total } & 16 & 0,948 & 0,948 \\
\hline
\end{tabular}

Se enfatizó que, el procedimiento de análisis de datos, se realizaron con el programa estadístico SPSS v.26, utilizado para todos los análisis estadísticos pertinentes a la investigación, expresado en tablas y gráfica; y, Microsoft Excel $365^{\circ}$ para los análisis financieros, análisis FODA y Cuadro de Mando Integral, extrapolando información financiera y diagnóstico situacional de la empresa desde el planeamiento hasta la evaluación mediante los ratios financieros. De esta manera, se podrá demostrar los resultados de forma observacional (estado de la empresa) y estadísticamente (percepción).

Como acto preventivo, se procedió aplicar la prueba de normalidad en base a los pre y post test, por análisis de dimensiones y totalidad, con el estadístico de Shapiro Wilk, el cual fue el más idóneo por el tipo de investigación. 
Tabla 3. Diferencia Post y Pre test sobre la aplicación de la Contabilidad Gerencial en cada dimensión y la variable toma de decisiones en una Microempresa de Lima Metropolitana.

Diferencias Post test - Pre test

Factores

\begin{tabular}{lllc}
\cline { 2 - 4 } & \multicolumn{1}{c}{ Estadístico } & $\mathrm{gl}$ & Sig. \\
\hline Decisión de Financiamiento & 0,899 & 9 & 0,247 \\
Decisión sobre Inversión & 0,933 & 9 & 0,510 \\
Decisión sobre Riesgo & 0,867 & 9 & 0,113 \\
Decisión sobre Rentabilidad & 0,776 & & \\
& & 9 & 0,011 \\
Toma de Decisiones & 0,948 & & \\
& & 9 & 0,671
\end{tabular}

En la Tabla 3, se presenta la prueba de normalidad de Shapiro Wilk, evaluados en la diferencia del post test respecto del pre test, donde se encontraron que, a un nivel de significancia de 0,01 , dichos puntajes representan una distribución normal al tener niveles de significancia mayores al valor establecido; por lo tanto, las comparaciones correspondientes se realizarán mediante la prueba "t de Student" para dos medidas pareadas, siendo el estadístico idóneo para corroborar que la contabilidad gerencial influencia directamente en la efectiva toma de decisiones de la micro entidad gráfica.

\section{Limitaciones del estudio}

La primera limitación fue tener acceso a la información contable y administrativa de la microempresa. La segunda fue aplicar la encuesta previa para saber el nivel de conocimiento que tenían los trabajadores sobre la toma de decisiones y herramientas financieras. La tercera fue establecer los días y horarios disponibles de los operarios y dueño de la empresa, para capacitarlos en las herramientas de la contabilidad gerencial para que puedan ayudar a tomar decisiones en base a datos cuantificados, siendo 04 sesiones por espacio de 01 semana. La cuarta fue sensibilizar y adaptar el cambio a los trabajadores, guiándolos en la estructuración de las herramientas financieras y demostrándoles cómo puede influenciar en los diversos tipos de toma de decisiones, asimismo, también les ayudaba en su declaración de impuestos. La quinta fue el compromiso de los trabajadores, debido a que los de la parte técnica, decían que no influenciaba esos conocimientos en sus actividades; sin embargo, se logró interiorizar que sus opiniones podrían mejorar y guiar a tomar una decisión positiva para la microempresa. Por último, el séptimo limite fue la disponibilidad de tiempo de los investigadores para realizar un seguimiento persistente.

\section{Resultados y discusión \\ Análisis de datos observacionales}

Como punto de partida, se realizó un diagnóstico organizacional en términos generales, realizando una herramienta administrativa conocida como la Matriz de Fortalezas, Oportunidades, Debilidades y Amenazas (FODA), para esquematizar los defectos y virtudes, internos y externos, de la microempresa gráfica. 
Tabla 4. Matriz FODA.

\begin{tabular}{|c|c|}
\hline Debilidades & Amenazas \\
\hline
\end{tabular}

- Costos volátiles.

- Alta rotación de personal.

- Inexistente planificación y control de utilidades.

- Servicio sin plataforma web.

- Política no inclusiva para la toma de decisiones.
- Recesión económica nacional.

- Política de confinamiento por salubridad.

- Variación de precios de los insumos.

- Innovación tecnológica.

- Incremento de competidores.

\begin{tabular}{|c|c|}
\hline Fortalezas & Oportunidades \\
\hline
\end{tabular}

- Experiencia en el rubro.

- Poder de negociación ante los clientes.

- Local de alta concurrencia pública.

- Personal multifuncional.

- Autofinanciación.
- Uso de redes sociales (marketing digital).

- Participación en licitaciones públicas.

- Inclusión de extranjeros como mano de obra

- Diversificación del servicio.

- Acceso a préstamos bancarios.
En relación con lo planteado en la matriz mostrada, se formulan las estrategias integradoras, juntando las potencialidades (fortalezas y oportunidades) con los riesgos (debilidades y amenazas), para poder afrontar los desafíos y generar cambios positivos para superar las limitaciones actuales que tiene la microempresa gráfica. Este planteamiento se asemeja a lo que describe en su libro Sánchez (2020), en donde para realizar el análisis FODA para la empresa McDonald's, las fases aplicadas en este proceso fueron: (i) Elegir la situación a analizar y el objetivo a alcanzar. (ii) Implementación de propio análisis FODA. (iii) Selección de las debilidades, fortalezas, oportunidades y amenazas más relevantes. (iv) Integración de la matriz FODA. Mostrando las mismas tendencias que la empresa a analizar (Tabla 5).

Según Rodríguez (2016), es necesario diseñar una metodología de diagnóstico o guía que permita identificar los problemas existentes, para ello debe disponer de herramientas apropiadas que le permitan detectar los errores y aplicar los correctivos adecuados, predecir el futuro y lograr una planeación más idónea. 
Tabla 5. Estrategias extrapoladas de la Matriz FODA.

\begin{tabular}{|c|c|c|}
\hline Interno & Fortalezas & Debilidades \\
\hline 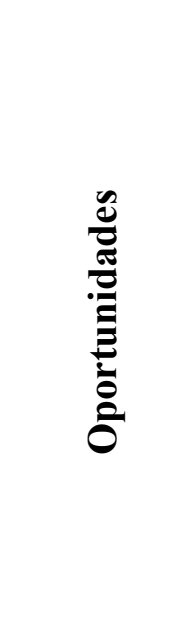 & $\begin{array}{l}\text { EFO1: Se utilizará la experiencia en el sector para } \\
\text { la promoción y comercialización mediante redes } \\
\text { sociales, empleando marketing digital. } \\
\text { EFO2: Se usará el poder de negociación ante los } \\
\text { clientes, para incursionar en licitaciones públicas. } \\
\text { EFO3: La organización empleará mano de obra } \\
\text { extranjera para ahorrar costos en pago de personal } \\
\text { para atender al público universitario y general. } \\
\text { EFO4: El personal multifuncional diversificará los } \\
\text { servicios al realizar "delivery", diseños } \\
\text { personalizados y pagos por transferencia bancaria. } \\
\text { EFO5: La condición financiera de la empresa } \\
\text { permitirá acceder a préstamos bancarios para las } \\
\text { inversiones a futuro ante contingencias. }\end{array}$ & $\begin{array}{l}\text { EDO1: El uso del marketing digital (redes } \\
\text { sociales) y estructuración de ingresos y egresos, } \\
\text { eliminará los costos volátiles. } \\
\text { EDO2: El personal solicitado en las licitaciones } \\
\text { públicas no se verá afectado debido a la necesidad } \\
\text { laboral de la mano de obra extranjera. } \\
\text { EDO3: La implementación del cuadro de mando } \\
\text { integral, permitirá revertir la planificación y } \\
\text { control inadecuados. } \\
\text { EDO4: La implementación del uso de plataformas } \\
\text { web permitirá conocer los servicios diversificados. } \\
\text { EDO5: La información financiera y perspectiva } \\
\text { operativa será determinante para incentivar la } \\
\text { política inclusiva en la toma de decisiones. }\end{array}$ \\
\hline 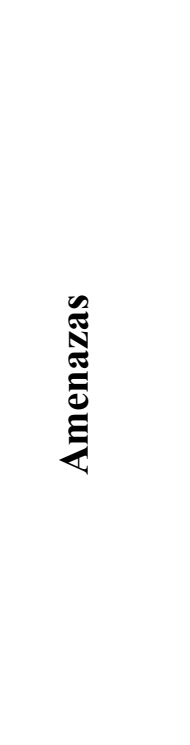 & $\begin{array}{l}\text { EFA1: La empresa usará la experiencia en el sector } \\
\text { para dinamizar y diversificar sus servicios para } \\
\text { soportar el efecto de la recesión económica del país. } \\
\text { EFA2: Se utilizará el poder de negociación para } \\
\text { reducir el alto riesgo de la inversión ante una política } \\
\text { de confinamiento por salubridad, en base a la } \\
\text { fidelización de sus clientes. } \\
\text { EFA3: La organización empleará su excelente } \\
\text { ubicación y clientes fidelizados, para concentrar el } \\
\text { servicio en ellos, para no verse afectado por la } \\
\text { variación de precios a nivel mundial. } \\
\text { EFA4: La cualidad multifuncional de los } \\
\text { trabajadores permitirán implementar y adaptarse a } \\
\text { las innovaciones tecnológicas pertinentes. } \\
\text { EFA5: La financiación permitirá sostener los } \\
\text { cambios de la diversificación de servicios para } \\
\text { diferenciarse ante el incremento de competidores. }\end{array}$ & $\begin{array}{l}\text { EDA1: La firma reducirá sus costos volátiles a } \\
\text { través de las innovaciones en los servicios para } \\
\text { afrontar la recesión económica nacional. } \\
\text { EDA2: Se disminuirá la rotación de personal en la } \\
\text { utilización de mano de obra extranjera ante la } \\
\text { política de confinamiento por salubridad. } \\
\text { EDA3: Las herramientas de la contabilidad } \\
\text { gerencial permitirá planificar y controlar las } \\
\text { utilidades en base a indicadores para aminorar el } \\
\text { impacto de la variación de precios por insumos. } \\
\text { EDA4: La utilización de la plataforma web será } \\
\text { motivo de muestra de la innovación tecnológica, } \\
\text { donde se usará plataformas de pago virtual, } \\
\text { maquinarias nuevas y catalogo "online". } \\
\text { EDA5: La inclusión de los trabajadores en la toma } \\
\text { de decisiones se sustenta para permitir ver y opinar } \\
\text { las deficiencias de los competidores en el mercado. }\end{array}$ \\
\hline
\end{tabular}

Al tener las estrategias complementadas, se procedió a implementar las herramientas de la contabilidad gerencial, adaptadas a la realidad de la microempresa gráfica: por ende, se estructuró los ingresos, costos y gastos en las matrices denominados Estados Financieros, donde se colocará los Estados de Situación Financiera (ex Balance General) y el Estado de Resultados (ex Estado de Ganancias y Pérdidas), realizando análisis vertical y horizontal; asimismo, se esquematizó los Ratios Financieros (Solvencia, Liquidez y Rentabilidad). 
Tabla 6. Estado de Situación Financiera de la microempresa gráfica Copy Data E.I.R.L. por el periodo $2019-2018$.

\begin{tabular}{|c|c|c|c|c|c|}
\hline$\underline{\text { Activo }}$ & $\begin{array}{c}\text { Valor } \\
\text { Histórico } \\
\text { al 31/12/2019 }\end{array}$ & $\underline{\%}$ & $\begin{array}{c}\text { Valor } \\
\text { Histórico } \\
\text { al } \mathbf{3 1 / 1 2 / 2 0 1 8}\end{array}$ & $\underline{\%}$ & $\begin{array}{c}\text { Aumentos y } \\
\text { disminuciones }\end{array}$ \\
\hline Activo Corriente & $\mathrm{S} /$ & & $\mathrm{S} /$ & & $\mathrm{S} /$ \\
\hline Efectivo y equivalente de efectivo & $111,145,00$ & $29 \%$ & $18,316,00$ & $6 \%$ & $92,829,00$ \\
\hline Cuentas por cobrar con terceros & $18,306,00$ & $5 \%$ & $9,300,00$ & $3 \%$ & $9,006,00$ \\
\hline Mercaderías (existencias) & $101,172,00$ & $26 \%$ & $152,350,00$ & $47 \%$ & $-51,178,00$ \\
\hline Productos terminados & $43,356,00$ & $11 \%$ & $33,800,00$ & $10 \%$ & $9,556,00$ \\
\hline Productos en Proceso & $20,600,00$ & $5 \%$ & $26,717,00$ & $8 \%$ & $-\quad 6,117,00$ \\
\hline Materias Primas & $40,000,00$ & $10 \%$ & $25,800,00$ & $8 \%$ & $14,200,00$ \\
\hline Envases y Embalajes & $5,600,00$ & $1 \%$ & 900,00 & $\mathbf{0 \%}$ & $4,700,00$ \\
\hline Existencias por Recibir & $14,411,00$ & $4 \%$ & $28,900,00$ & $9 \%$ & $-14,489,00$ \\
\hline Total Activo Corriente & $354,590,00$ & $92 \%$ & $296,083,00$ & $91 \%$ & $58,507,00$ \\
\hline Activo No Corriente & $\mathrm{S} /$ & & $\mathrm{S} /$ & & $\mathrm{S} /$ \\
\hline Inmuebles, Maquinaria y Equipo & $42,000,00$ & $11 \%$ & $41,254,00$ & $13 \%$ & 746,00 \\
\hline Dep. Inm. Activo Arrend. Financ. acum. & $-19,001,00$ & $-5 \%$ & $-14,857,00$ & $-5 \%$ & $-4,144,00$ \\
\hline Activo Diferido & $7,660,00$ & $2 \%$ & $1,634,00$ & $1 \%$ & $6,026,00$ \\
\hline Total Activo No Corriente & $30,659,00$ & $8 \%$ & $28,031,00$ & $9 \%$ & $2,628,00$ \\
\hline Total activo neto & $385,249,00$ & $100 \%$ & $324,114,00$ & $100 \%$ & $61,135,00$ \\
\hline
\end{tabular}

\section{Pasivo}

Pasivo Corriente

S/ S/

\begin{tabular}{|c|c|c|c|c|c|}
\hline Trib. y Aport. Sist.pens. y salud por pagar & $10,436,00$ & $3 \%$ & $10,650,00$ & $3 \%$ & $-214,00$ \\
\hline Remuneraciones y participaciones por pagar & $4,250,00$ & $1 \%$ & $2,420,00$ & $1 \%$ & $1,830,00$ \\
\hline Ctas p. Pagar Comerciales - terceros & $64,778,00$ & $17 \%$ & $63,056,00$ & $19 \%$ & $1,722,00$ \\
\hline Total Pasivo Corriente & $79,464,00$ & $21 \%$ & $76,126,00$ & $23 \%$ & $3,338,00$ \\
\hline Pasivo No Corriente & \multicolumn{2}{|l|}{$\mathrm{S} /$} & \multicolumn{2}{|l|}{$\mathrm{S} /$} & $\mathrm{S} /$ \\
\hline Obligaciones Financieras & $32,937,00$ & $9 \%$ & $111,582,00$ & $34 \%$ & - $78,645,00$ \\
\hline Total Pasivo No Corriente & $32,937,00$ & $9 \%$ & $111,582,00$ & $34 \%$ & - 78,645,00 \\
\hline Total Pasivo & $112,401,00$ & $29 \%$ & $187,708,00$ & $58 \%$ & - 75,307,00 \\
\hline \multicolumn{3}{|l|}{ Patrimonio } & \multicolumn{2}{|l|}{$\mathrm{S} /$} & \\
\hline Capital & $36,861,00$ & $10 \%$ & $36,861,00$ & $11 \%$ & 0 \\
\hline Resultados Acumulados Positivo & $99,545,00$ & $26 \%$ & $58,396,00$ & $18 \%$ & $41,149,00$ \\
\hline Utilidad del Ejercicio & $136,442,00$ & $35 \%$ & $41,149,00$ & $13 \%$ & $95,293,00$ \\
\hline Total Patrimonio & $272,848,00$ & $71 \%$ & $136,406,00$ & $42 \%$ & $136,442,00$ \\
\hline Total Pasivo y Patrimonio & $385,249,00$ & $100 \%$ & $324,114,00$ & $100 \%$ & $61,135,00$ \\
\hline
\end{tabular}


En base al análisis del Estado de Situación Financiera, se puede inferir a nivel de Activos, que del año 2018 al 2019, se mejoró la capacidad de obtener efectivo, debido a que las políticas de cobranzas fueron más estrictas al reducir el plazo de cobros pendientes e implementar la opción de pago por transferencia interbancaria; además, de realizar seguimientos a los pagos por entregables por las licitaciones que tienen con el estado por la prestación de su servicio. Asimismo, se refleja la reducción del monto de mercadería y productos en proceso, así como, aumento de los productos terminados, ya que los trabajos están realizándose en función al tiempo del pedido y/o entregables, teniendo una pequeña reserva ante una entrega fallida. La materia prima y embalaje se incrementaron y las existencias disminuyen, debido a que se aumentó la producción por los pedidos de los diversos clientes del sector público y privado. La empresa renovó dos maquinarias para mejor desempeño y automatizado de impresión láser, por ello, se incrementó los activos no corrientes y se instauró el termino de depreciación de maquinaria por año, mostrando valores reales de activos comparando el año 2019 con la adaptación del 2018.

A nivel de pasivos, se disminuyó el pago de tributos debido a la compra de maquinarias y materia prima el cual reduce el pago de impuestos; a su vez, por la mayor cantidad de contratos realizados, se tuvo que emplear mayor materia prima y recurso humano, teniendo que pagar horas extras, incrementando el gasto por remuneraciones y las cuentas por pagar comerciales, para solventar la compra de materia prima mediante créditos y pagos a cuotas; sin embargo, por la cultura de autofinanciación que tenía la entidad, se pudo amortizar la deuda en varios plazos, mostrando una reducción en las obligaciones financieras en el 2019 respecto al 2018.

A nivel de Patrimonio, no se invirtió el mismo capital propio, con la particularidad de que se logró mayor utilidad diferencial en el 2019 respecto al 2018, del cual fue de S/ 95293,00 , a nivel contable y correctamente estructurado, aplicando las estrategias propuestas como alternativa de solución y teniendo conocimiento de los costes y de sus ingresos y gastos esquematizados, teniendo en consideración como información observable para una efectiva toma de decisiones en la microempresa gráfica.

Sin embargo, para tener una visión más precisa de los ingresos, gastos y costes, se procedió a estructurar y analizar el Estado de Resultados de la micro entidad gráfica, como muestra de datos fidedignos y posterior declaración ante la SUNAT. 
Tabla 7. Estado de Resultados de la microempresa gráfica Copy Data E.I.R.L. por el periodo $2019-2018$.

\begin{tabular}{|c|}
\hline Valor \\
Histórico \\
al \\
$31 / 12 / 2019$ \\
\hline
\end{tabular}

S/

\begin{tabular}{clr}
\hline \multicolumn{1}{c|}{ VENTAS NETAS } & $629,420,00$ \\
\cline { 2 - 3 } ( - ) & Costo de Ventas & $340,500,00$ \\
\cline { 2 - 3 } ( ) & Gastos de Venta & $288,920,00$ \\
\cline { 2 - 3 } & Gastos de Administración & $38,006,00$ \\
\hline & Utilidad o Pérdida Operativa & $40,479,00$ \\
\hline
\end{tabular}

\begin{tabular}{|c|c|c|}
\hline$(-)$ & Gastos Financieros & $27,900,00$ \\
\hline \multirow[t]{2}{*}{+} & Ingresos Financieros Gravados & 0 \\
\hline & Otros Ingresos gravados & $11,000,00$ \\
\hline+ & Otros Ingresos no gravados & 0 \\
\hline+ & $\begin{array}{l}\text { Enajenación de valores y bienes del } \\
\text { activo fijo }\end{array}$ & 0 \\
\hline$(-)$ & $\begin{array}{l}\text { Costo enajenación de valores y } \\
\text { bienes Activo Fijo }\end{array}$ & 0 \\
\hline \multirow[t]{2}{*}{$(-)$} & Gastos Diversos & 0 \\
\hline & $\begin{array}{l}\text { Utilidad o Perdida antes de } \\
\text { participaciones }\end{array}$ & $193,535,00$ \\
\hline
\end{tabular}

$(-)$

\begin{tabular}{c|r|}
\hline $\begin{array}{l}\text { Distribución Legal de la Renta } \\
\text { (Part. Trabajadores) }\end{array}$ & 0 \\
\hline $\begin{array}{c}\text { Utilidad o Perdida antes de } \\
\text { Impuestos }\end{array}$ & $193,535,00$ \\
\hline Impuesto a la Renta & $57,092,83$ \\
\hline Utilidad o Perdida del Ejercicio & $136,442,18$ \\
\hline
\end{tabular}
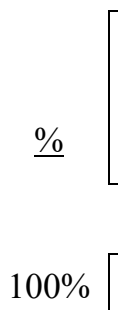

$100 \%$
$-54 \%$
$46 \%$
$-6 \%$
$-6 \%$
$33 \%$

$-4 \%$
$0 \%$
$2 \%$
$0 \%$
$0 \%$
$0 \%$
$0 \%$
$31 \%$

\begin{tabular}{|c|}
\hline Valor \\
Histórico \\
al \\
$31 / 12 / 2018$ \\
\hline
\end{tabular}

$\mathrm{S} /$

\begin{tabular}{|c|c|}
\hline $376,460,00$ & $100 \%$ \\
\hline $236,421,00$ & $-63 \%$ \\
\hline $140,039,00$ & $37 \%$ \\
\hline $34,554,00$ & $-9 \%$ \\
\hline $39,946,00$ & $-11 \%$ \\
\hline $65,539,00$ & $17 \%$ \\
\hline
\end{tabular}

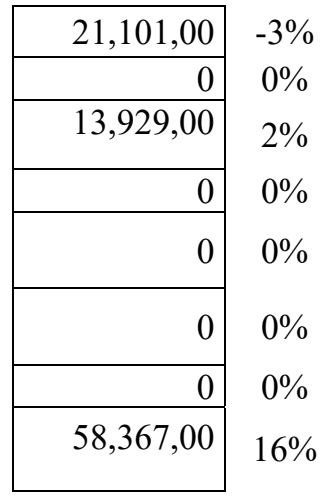

AUMENTOS Y DISMINUCIONES

$\mathrm{S} /$

\begin{tabular}{|r|}
\hline $252,960,00$ \\
\hline $104,079,00$ \\
\hline $148,881,00$ \\
\hline $3,452,00$ \\
\hline 533,00 \\
\hline $144,896,00$ \\
\hline
\end{tabular}

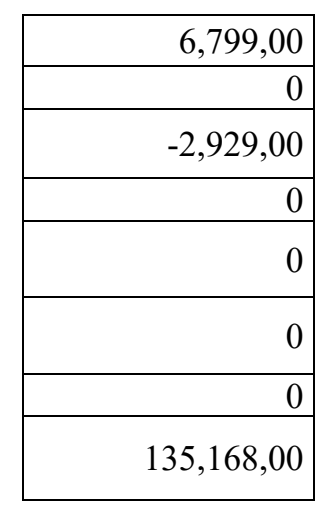

Al analizar el Estado de Resultados, se denota que, en todos los ámbitos de Utilidades o Pérdidas, se obtuvo ganancias con diferencias significativas en el año 2019 respecto al 2018; por lo tanto, se demuestra que con la organización y planificación desde el principio se puede obtener mayores utilidades con la misma cantidad de capital propio invertido, al utilizar créditos bancarios para compras.

En la micro entidad no se realiza repartición de utilidades debido a la política del gobierno peruano, de que las microempresas no están sujetas a ese criterio por el monto de ingresos que se percibe; asimismo, al ser local alquilado, no se tiene costo de enajenación, es por ello, que no tienen valor en el instrumento esquematizado. Se debe enfatizar que no se incluyó el costo de asesoramiento, debido a que fue un compromiso de los investigadores con la microempresa gráfica. En función a estos resultados, Ortega (2020) describe que el Estado de Resultados es una herramienta que nos permite calcular las pérdidas y ganancias generadas por la empresa, mostrando ingresos $\mathrm{y}$ gastos incurridos durante un período determinado. Su estructura se ajusta al principio de devengo. Esto significa que los ingresos y gastos deben estimarse en función 
del flujo real de bienes y servicios, es decir, cuando una empresa entrega productos o brinda servicios a los clientes, independientemente del tiempo que tarde en producir dinero, o, del flujo de fondos, es decir, recibos y pagos.

Para un mejor entendimiento del directivo y de los trabajadores, se implementó el uso de los ratios financieras, para que tengan un panorama general del estado financiero de la empresa. Para la formulación de las ratios, se parte del Estados de Situación Financiera y del Estado de Resultados.
Según Diaz y Dextre (2017), los ratios financieros son el cociente entre dos órdenes de magnitud, lo que puede hacer que la información obtenida sea mejor para estudiar cada dato por separado. Por supuesto, puede comparar los estados situación financiera, el estado de resultados y/o los elementos del estado de situación financiera con el estado de resultados. Por ende, son solo una forma útil de resumir una cantidad masiva de información y comparar el comportamiento de las empresas.

Tabla 8. Ratios Financieros de la microempresa gráfica Copy Data E.I.R.L.

\begin{tabular}{|c|c|c|c|c|c|}
\hline \multicolumn{6}{|c|}{ Ratios de liquidez } \\
\hline Tipo & \multicolumn{2}{|c|}{ Al 31/12/2019 } & Índice & Al 31/12/2018 & Índice \\
\hline Liquidez General & $\frac{\text { Activo Corriente }}{\text { Pasivo Corriente }}$ & $\frac{354,590,00}{79,464,00}$ & 4,46 & $\frac{296,083,00}{111,582,00}$ & 2,65 \\
\hline $\begin{array}{l}\text { Liquidez Severa } \\
\text { (Prueba Ácida) }\end{array}$ & $\frac{\text { Activo Corriente-Existencias }}{\text { Pasivo Corriente }}$ & $\frac{354,590,00-101,172,00}{79,464,00}$ & 3,19 & $\frac{296,083.00-152,350,00}{111,582,00}$ & 1,89 \\
\hline $\begin{array}{l}\text { Liquidez Absoluta } \\
\text { (Efectividad) }\end{array}$ & $\frac{\text { Efectivo y Equivalente }}{\text { Pasivo Corriente }}$ & $\frac{111,145,00}{79,464,00}$ & 1,40 & $\frac{18,316,00}{111,582,00}$ & 0,16 \\
\hline \multicolumn{6}{|c|}{ Ratios de Solvencia } \\
\hline Tipo & \multicolumn{2}{|c|}{ Al 31/12/2019 } & $\%$ & Al 31/12/2018 & $\%$ \\
\hline $\begin{array}{l}\text { Solvencia de } \\
\text { Activo }\end{array}$ & $\frac{\text { Pasivo Total }}{\text { Activo Total }}$ & $\frac{112,401,00}{385,249,00}$ & $29 \%$ & $\frac{187,708,00}{324,114,00}$ & $58 \%$ \\
\hline $\begin{array}{l}\text { Solvencia de } \\
\text { Patrimonio }\end{array}$ & $\frac{\text { Pasivo Total }}{\text { Patrimonio }}$ & $\frac{112,401,00}{272,848,00}$ & $41 \%$ & $\frac{187,708,00}{136,406,00}$ & $138 \%$ \\
\hline \multicolumn{6}{|c|}{ Ratios de Rentabilidad } \\
\hline Tipo & \multicolumn{2}{|c|}{ Al 31/12/2019 } & $\%$ & Al 31/12/2018 & $\%$ \\
\hline $\begin{array}{l}\text { Rentabilidad de } \\
\text { Utilidad Neta }\end{array}$ & $\frac{\text { Utilidad del Ejercicio }}{\text { Ventas Netas }}$ & $\frac{136,442,18}{629,420,00}$ & $22 \%$ & $\frac{41,148,74}{376,460,00}$ & $11 \%$ \\
\hline $\begin{array}{l}\text { Rentabilidad Sobre } \\
\text { Patrimonio } \\
\text { (ROE) }\end{array}$ & $\frac{\text { Utilidad del Ejercicio }}{\text { Patrimonio }}$ & $\frac{136,442,18}{272,848,00}$ & $50 \%$ & $\frac{41,148,74}{136,406,00}$ & $30 \%$ \\
\hline $\begin{array}{c}\text { Rentabilidad de } \\
\text { Activos Totales } \\
\text { (ROA) }\end{array}$ & $\frac{\text { Utilidad antes de Impuestos }}{\text { Activo Total }}$ & $\frac{193,535,00}{385,249,00}$ & $50 \%$ & $\frac{58,367,00}{324,114,00}$ & $18 \%$ \\
\hline $\begin{array}{l}\text { Rentabilidad Sobre } \\
\text { Inversión } \\
\text { (ROI) }\end{array}$ & $\frac{\text { Utilidad Ejercicio - Inversión }}{\text { Inversión* }}$ & $\frac{136,442.18-36,861,00}{36,861,00}$ & $270 \%$ & $\frac{41,148.74-36,861,00}{36,861,00}$ & $12 \%$ \\
\hline
\end{tabular}


Los ratios de liquidez se relaciona directamente con la capacidad de pago de deudas que tiene una empresa de cualquier nivel, teniendo en consideración de que si supera al valor " 1 " es considerado ligeramente estable económicamente, caso contrario, es decir menor a " -1 ", es decir la entidad no tiene consistencia de pago estable; sin embargo, para el presente estudio, en el año 2019, tras la implementación de la contabilidad gerencial y las estrategias planificadas, se demuestra que se mejoró notablemente la sostenibilidad financiera, teniendo índices que se incrementaron más del $50 \%$ en sus diversos tipos, comparándolo con el año 2018, superando inclusive la "prueba acida" y la "efectividad de liquidez", mostrando un excelente estado de liquidez que tiene la microempresa, al tener capacidad de pagos de deudas financieras para amortizarla en corto plazo. Los ratios de solvencia indican la capacidad de endeudamiento que tiene una entidad empresarial; es decir nivel de compromiso de los activos y/o patrimonio respecto a los pasivos; para el caso de Copy Data E.I.R.L., en el 2018 mostró una insolvencia financiera al superar el $50 \%$, pero, al implementar la solución metodológica, se pudo incrementar los activos y patrimonio, así como, reducir los pasivos, hasta lograr $20 \%$ teniendo una muy buena solvencia financiera en el año 2019; por ende, se puede conseguir créditos bancarios con facilidad para reinvertir y obtener mayores beneficios. Los ratios de rentabilidad muestra la capacidad de la organización en generar utilidades, por ejemplo, en el ratio de utilidades netas, el porcentaje se duplicó en el año 2019 respecto del 2018, lo cual refleja mayor ganancia para el directivo. Asimismo, en el ratio de rentabilidad sobre patrimonio (ROE), mejoró en un $20 \%$, debido al incremento en las utilidades en el ejercicio a pesar de no haber aumentado en la inversión de capital propio, mediante la financiación bancaria, mostrando una sostenibilidad financiera. Además, la Rentabilidad sobre los activos totales (ROA), aproximadamente se triplico en porcentaje en el 2019 respecto al 2018, debido al aumento del valor de los activos que tiene la microempresa gráfica, tanto en bienes como en efectivo, lo cual es un indicador fundamental al momento de solicitar un préstamo en una entidad financiera. Cabe mencionar que, la Rentabilidad sobre las inversiones (ROI), se incrementó exponencialmente a $270 \%$ en el 2019 comparando con el $12 \%$ del 2018, siendo un excelente indicador para el inversionista (gerente), debido a que fue una excelente inversión y riesgo el que tomó al adoptar las medidas y soluciones que la contabilidad gerencial ofrece al tomar decisiones.

Según Parraga et al. (2021), el 76,7\% de los autores estudiados (13 artículos) concibe indicadores de gestión financiera asociados fundamentalmente con razones o ratios específicamente financieros, referidos fundamentalmente con liquidez, solvencia, eficiencia, actividad, endeudamiento y rentabilidad.

Buele, et al. (2020), concluye que aplicadas las razones financieras a las compañías auditadas estudiadas como el grupo control y las compañías no auditadas estudiadas como el grupo control, se observan niveles de liquidez mucho más favorables en las compañías no auditadas. La razón del efectivo (efectivo/pasivo corriente) de las compañías no auditadas $(1,98)$ supera por más del doble al resultado obtenido por las empresas auditadas $(0,64)$; sin embargo, razones financieras como la prueba ácida y la razón del inventario al pasivo corriente demuestran que este efectivo no se encuentra concentrado en los inventarios, y un $45 \%$ de las empresas no auditadas concentran sus deudas al corto plazo.

Se enfatiza que, como herramienta de evaluación, se implementó el Cuadro de Mando Integral (Balanced Scorecard), para poder visualizar el estado financiero, operativo, administrativo y de innovación, en tiempo real y en base a cuatro (04) perspectivas que dinamiza e integra las falencias con 
las fortalezas que tiene una empresa. Para este caso, lo adaptamos a la realidad de la microempresa gráfica; $y$, para tener los resultados de los indicadores, se extrapoló de los reportes de gestión y de la base de datos que registra la empresa de los clientes $\mathrm{y}$ actividades programadas. Cabe mencionar que las variaciones estarán representadas en flechas direccionadas, $\mathrm{y}$, si no existe variación, en un círculo

Tabla 9. Ratios Financieros de la microempresa gráfica Copy Data E.I.R.L.

\begin{tabular}{|c|c|c|c|c|}
\hline PERSPECTIVA FINANCIERA & 31/12/2019 & $31 / 12 / 2018$ & Variación & Situación \\
\hline $\begin{array}{l}\text { ROE-Rendimiento sobre el Patrimonio } \\
\text { Utilidad Neta/Patrimonio }\end{array}$ & $50,01 \%$ & $30,17 \%$ & & Bueno \\
\hline $\begin{array}{l}\text { ROA-Rendimiento de los Activos } \\
\text { Utilidad Neta/Activo Total }\end{array}$ & $50,24 \%$ & $18,01 \%$ & & Bueno \\
\hline EVA-Valor Económico Agregado & $99,581,18$ & $4,287,74$ & & Bueno \\
\hline $\begin{array}{l}\text { Crecimiento de las Ventas Netas } \\
\text { (Ventas Netas 2/Ventas Netas 1)-1 }\end{array}$ & $67,20 \%$ & $24,29 \%$ & & Bueno \\
\hline PERSPECTIVA DEL CLIENTE & $31 / 12 / 2019$ & $31 / 12 / 2018$ & Variación & Situación \\
\hline $\begin{array}{l}\text { Ratio de Ventas por Cliente } \\
\text { Ventas Netas/Total de Clientes }\end{array}$ & $5,619,82$ & $4,327,13$ & & Bueno \\
\hline $\begin{array}{l}\text { Ratio de Crecimiento de Clientela } \\
\text { Clientes Nuevos/Clientes Totales }\end{array}$ & $22,32 \%$ & $9,47 \%$ & & Bueno \\
\hline $\begin{array}{l}\text { Ratio de Quejas de los clientes } \\
\mathrm{N}^{\circ} \text { Quejas/Total de Clientes }\end{array}$ & $37,81 \%$ & $79,67 \%$ & & Bueno \\
\hline $\begin{array}{c}\text { PERSPECTIVA DE APRENDIZAJE } \\
\text { Y CRECIMIENTO }\end{array}$ & $31 / 12 / 2019$ & 31/12/2018 & Variación & Situación \\
\hline $\begin{array}{l}\text { Ratio de Ventas por Empleado } \\
\text { Ventas Netas/Número de Empleados }\end{array}$ & $69,935,56$ & $41,828,90$ & & Bueno \\
\hline $\begin{array}{l}\text { Ratio de Utilidad por Trabajador } \\
\text { Utilidad Neta/Número de Empleados }\end{array}$ & $15,160,24$ & $4,572,08$ & & Bueno \\
\hline $\begin{array}{l}\text { Ratio de Grado de Satisfacción } \\
\mathrm{N}^{\circ} \text { Quejas/Total de Empleados }\end{array}$ & $44,45 \%$ & $22,23 \%$ & & Malo \\
\hline $\begin{array}{l}\text { Ratio de Trabajadores Capacitados } \\
\mathrm{N}^{\circ} \text { Trab.Capa./Total de Empleados }\end{array}$ & $100 \%$ & $0 \%$ & & Bueno \\
\hline
\end{tabular}


Implementación de la contabilidad gerencial para la efectiva toma de decisiones en una microempresa gráfica de Lima Metropolitana

\begin{tabular}{lllll|}
\hline $\begin{array}{c}\text { PERSPECTIVA DE PROCESOS } \\
\text { INTERNOS }\end{array}$ & $\mathbf{3 1 / 1 2 / 2 0 1 9}$ & $\mathbf{3 1 / 1 2 / 2 0 1 8}$ & Variación & Situación \\
\hline Ratio de Costo de Mercadería & 11,10 & 8,43 & Malo \\
Costo de Mercadería/Activo Fijo & & & Bueno \\
\hline Ratio Rendimiento de Activo Fijo & 20,52 & 13,43 & Bueno \\
Unidades vendidas/Activo Fijo & $0 \%$ & $40 \%$ & Bueno \\
\hline Ratio de Eficiencia de la Inspección \\
$\begin{array}{l}\text { Unid. Defec./Total Unid.Adq. } \\
\text { Ratio de Innovación } \\
\text { Ventas Nuevo Produc./Total de Ventas }\end{array}$ \\
\hline
\end{tabular}

Al evaluarlas cuatro (04) perspectivas del cuadro de mando integral, comparamos los resultados del año 2019 y 2018, donde denotamos que ahora existe un excelente y consistente sistema financiero interno, debido a que en todos los ratios se mostró un incremento de las utilidades e ingresos, relevando una variación positiva en todos sus ámbitos. Respecto a los usuarios externos (clientes), demuestra una mejora notable, inclusive obteniendo mayor clientela y reduciendo el porcentaje de quejas, dio prestigio en el rubro a la microempresa gráfica. Además, los usuarios internos (empleados) favorecieron el incremento de las utilidades por empleado; así como, de capacitar a la totalidad de los empleados en temas de herramientas de contabilidad gerencial y de tecnologías, al adquirir 2 nuevas maquinarias para repotenciar la productividad al tener nuevos clientes, alcanzando un aprendizaje que estaba descuidado durante año; sin embargo, se obtuvo un aumento en el porcentaje de insatisfacción de los empleados, debido a la resistencia al cambio que tenían en sus estándares de trabajos. Los procesos internos reflejaron un incremento en el costo de mercadería con la finalidad de tener mayores utilidades, el cual implica mayor gasto, el cual la brecha fue financiada por un ente bancario; ergo, respecto a los activos e innovaciones, se tuvo incrementos de variación positiva, demostrando de esta manera, una efectiva toma de decisiones a nivel global en la evaluación organizacional, teniendo que mejorar en dos criterios que a largo plazo se puede superar. Los presentes resultados son semejantes y demuestran lo que dice Sanna (2018); ya que, al establecer una serie de indicadores, el Balanced Scorecard brinda una perspectiva global sobre los objetivos y estrategias de la empresa a corto, mediano y largo plazo. Estos se cuantifican y miden los avances de proyectos y tareas de la empresa. Los elementos innovadores de esta herramienta de gestión se pueden encontrar en el análisis basado en cuatro dimensiones: (i) El punto de vida financiero. (ii) La dimensión humana. (iii) Los procesos organizativos internos. (iv) La dimensión aprendizaje, crecimiento e innovación.

\section{Análisis estadístico de datos}

En base a Millones et al. (2016), con la finalidad de medir la percepción sobre la influencia de la contabilidad gerencial en la toma de decisiones en la microempresa gráfica de Lima Metropolitana, se utilizó de la prueba $t$ de Student para dos medidas pareadas, bajo un nivel de significancia de 0,01 ; con la comparación de las medidas pre y post test tomados, que describe el antes y después de la 
implementación de la contabilidad gerencial. Cañizares y Jurado (2019), entre los resultados obtenidos, fue posible evidenciar la necesidad de una impronta significativa de las investigaciones relacionadas con la contabilidad de gestión y su integración con nuevos paradigmas contables.

Cedeño et al. (2019), señala que la aplicación de estrategias adecuadas en el ámbito económico-financiero, las Pymes podrán mantenerse en la línea del tiempo, ser más competitivas, lograr un adecuado equilibrio entre el riesgo y la rentabilidad que les permita generar valor agregado que maximice sus rendimientos y permita el logro de los objetivos estratégicos de las mismas.
Wasbaldo (2018), manifiesta que los resultados permiten confirmar que existe correlación entre la contabilidad gerencial y toma de decisiones, la misma que es expresada a través del coeficiente de correlación Rho de Spearman para el que toma el valor de 0,746, lo cual indica que cuanto mejor se realice la contabilidad gerencial, mejor se toma de decisiones.

Tanta (2020), concluye que la falta de conocimiento en el tema de la planificación estratégica con el apoyo fundamental de la contabilidad gerencial, hace de las empresas de telefonía móvil pierdan oportunidades de poder crecer empresarialmente.

Tabla 10. Comparación entre Pre y Post aplicación de la contabilidad gerencial en la toma de decisiones en una microempresa gráfica de Lima Metropolitana.

\begin{tabular}{|c|c|c|c|c|c|c|c|c|}
\hline \multirow{3}{*}{ Factores } & \multicolumn{8}{|c|}{ Diferencias Post test - Pre test } \\
\hline & \multirow{2}{*}{ Media } & \multirow{2}{*}{ SD } & \multirow{2}{*}{$\mathrm{EE}$} & \multicolumn{2}{|c|}{$95 \% \mathrm{IC}$} & \multirow{2}{*}{$\mathrm{T}$} & \multirow{2}{*}{$\mathrm{gl}$} & \multirow{2}{*}{ Sig. } \\
\hline & & & & Inferior & Superior & & & \\
\hline Toma de Decisiones & 16,889 & 4,457 & 1,486 & 13,463 & 20,315 & 11,369 & 9 & 0,000 \\
\hline
\end{tabular}

La Tabla 10, muestra el estadístico de contraste " $t$ " $=11,369$ y $\mathrm{p}$-valor $=0,000$ que es menor al " $t$ " tabular $=1,96$ y al nivel de significancia $=$ 0,01 ; en consecuencia, se puede afirmar que existen diferencias altamente significativas entre Pre y Post aplicación de la Contabilidad Gerencial en la toma de decisiones. Además, la diferencia promedio obtenido con un 95\% de confianza entre el post y pretest se encuentra el rango de [13,463 - 20,315]; por ende, se puede confirmar que la aplicación de la contabilidad gerencial en una microempresa gráfica de lima metropolitana permite tomar de decisiones efectivas.

Tabla 11. Comparación entre Pre y Post aplicación de la Contabilidad Gerencial en cada dimensión de la toma de decisiones en una microempresa gráfica.

\begin{tabular}{|c|c|c|c|c|c|c|c|c|}
\hline \multirow{3}{*}{ Factores } & \multicolumn{8}{|c|}{ Diferencias Post test - Pre test } \\
\hline & \multirow{2}{*}{ Media } & \multirow{2}{*}{$\mathrm{SD}$} & \multirow{2}{*}{$\mathrm{EE}$} & \multicolumn{2}{|c|}{$95 \% \mathrm{IC}$} & \multirow{2}{*}{$\mathrm{t}$} & \multirow{2}{*}{$\mathrm{gl}$} & \multirow{2}{*}{ Sig. } \\
\hline & & & & Inferior & Superior & & & \\
\hline Decisión de Financiamiento & 5,000 & 2,958 & 0,986 & 2,726 & 7,274 & 5,071 & 9 & 0,001 \\
\hline Decisión sobre Inversión & 4,667 & 1,732 & 0,577 & 3,335 & 5,998 & 8,083 & 9 & 0,000 \\
\hline Decisión sobre Riesgo & 4,111 & 1,364 & 0,455 & 3,062 & 5,160 & 9,041 & 9 & 0,000 \\
\hline Decisión sobre Rentabilidad & 3,111 & 1,167 & 0,389 & 2,214 & 4,008 & 8,000 & 9 & 0,000 \\
\hline
\end{tabular}




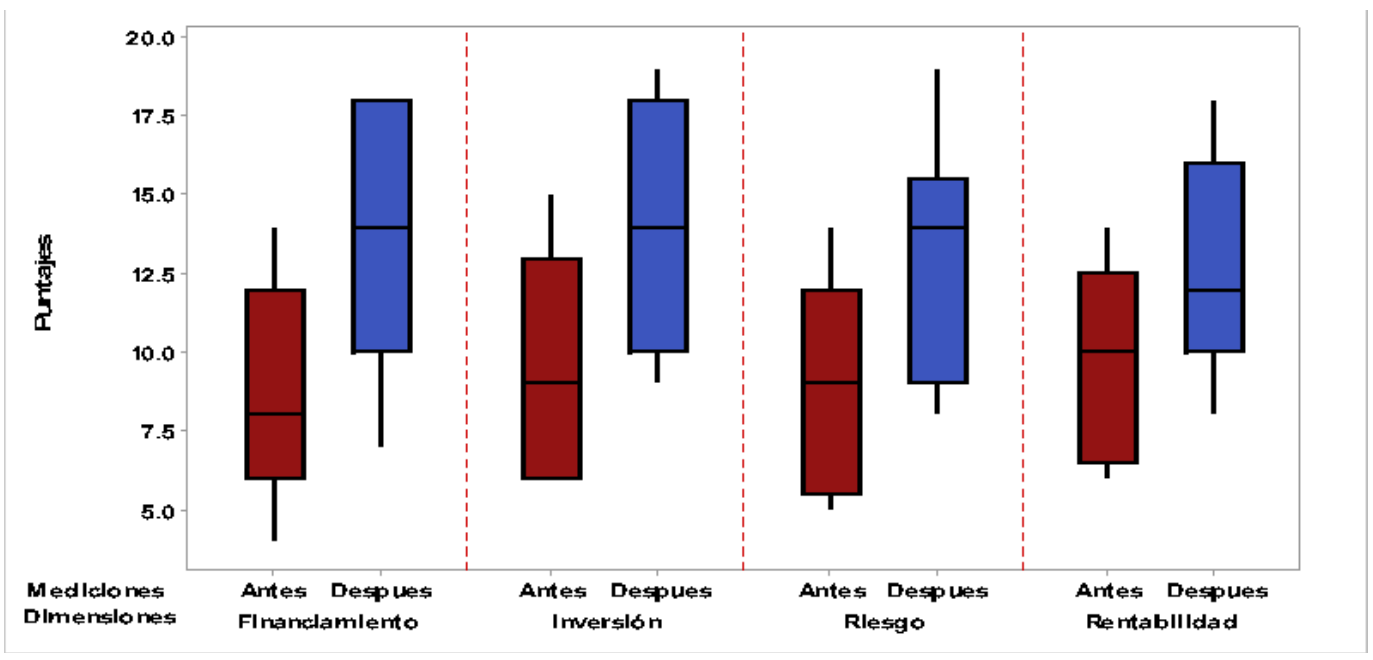

Figura 1. Diagrama de cajas y bigotes Pre y Post aplicación de la Contabilidad Gerencial en las dimensiones de la toma de decisiones en una microempresa gráfica.

En la Tabla 11, se muestra el estadístico de contraste " $t>5$ " y p-valor $>0,001$, menor al $\mathrm{t}$ tabular $=1,96 \mathrm{y}$ al nivel de significancia $=$ 0,01 ; en consecuencia, se puede aseverar que existen diferencias altamente significativas entre Pre y Post aplicación de la Contabilidad Gerencial por cada dimensión de la Toma de Decisiones. Asimismo, la diferencia promedio obtenido con un $95 \%$ de confianza entre el post y pretest en cada dimensión es $>0$; por lo tanto, se puede cerciorar que la aplicación de la contabilidad gerencial en una microempresa gráfica de Lima Metropolitana tiene un efecto positivo en cada dimensión de la toma de decisiones, el cual se visualiza de mejor manera en la Figura 1.

\section{Conclusiones}

Se demostró que la contabilidad gerencial influye directamente en la efectividad de la toma de decisiones en la microempresa gráfica de Lima Metropolitana, por exponer información administrativa y operacional, especialmente, información financiera, con la finalidad de invertir y obtener mayores utilidades; para ello, se comprobó y cercioró, en base a las perspectivas de datos observacionales y análisis estadístico, la amplia ventaja que se obtiene al implementar las herramientas de la contabilidad gerencial para la toma de decisiones empresariales, reflejando beneficios económicos y sociales, siendo percibidos por los clientes internos y externos de la empresa. Sin embargo, para sustentar lo descrito, se analizaron de forma observacional los estados de situación financiera, estados de resultados, ratios financieros y cuadro de mando integral, mediante reportes de gestión estructurado durante el estudio y del cual se tuvo brechas positivas; y, asimismo, para validar la percepción de los miembros de la entidad sobre la efectividad de la toma de decisiones, se realizó un pre y post test, del cual validaba, estadísticamente, una muy positiva percepción sobre la implementación de la contabilidad gerencial en la microempresa gráfica y que se toma decisiones empleando metodologías que científicamente se comprobó su efectividad. Por ende, este trabajo pretende ser modelo para otras microempresas de diversos rubros que merecen ser estudiadas e impulsadas por los investigadores, permitiendo conocer la problemática y cerciorando la flexibilidad de la metodología de la contabilidad gerencial, al adaptarse a la realidad de cada organización, según su línea de negocio. 


\section{Literatura citada}

Buele, I.; Cuesta, F. \& Chillogalli, C. 2020. Métricas para el diagnóstico de los estados financieros de las compañías del sector industrial manufacturero en Cuenca, Ecuador. Innovar (online). Vol.30 (76); 63-75. Bogotá. Colombia.

http://www.scielo.org.co/scielo.php?pi$\mathrm{d}=\mathrm{S} 0121-50512020000200063 \&$ script $=$ sci abstract\&tlng=es

Cedeño, A.; Asencio, L. \& Villegas, M. 2019. Las estrategias gerenciales como base fundamental para la administración en los negocios. Revista Universidad y Sociedad. Vol. 11 (5): 191-200. Guayaquil. Ecuador. http://scielo.sld.cu/scielo. php script $=$ sci_abstract\&pid $=$ S22 18 36202019000500191\&lng=es\&nrm=iso

Cañizares, M.\& Jurado, O. 2019. Las publicaciones sobre la contabilidad de gestión con enfoque de ecoeficiencia: un desafío al futuro. Cofin Habana, 13 (1): 1-11. Cuba. http://scielo.sld.cu/scielo.php?script=sci arttext\&pid=S2073-60612019000100001

Díaz, O. \& Dextre, J. 2017. Libro: Ciencia Contable: Visión y Perspectiva. Editorial: Fondo Editorial PUCP. Perú. 415 p.

Flores, J. 2019. Libro: Contabilidad Gerencial: Contabilidad de Gestión Empresarial. Editorial: CECOF ASESORES E.I.R.L. Perú. 600 p.

Hernández - Sampieri, R.; Mendoza C. 2018. Libro: Metodología de la Investigación - Las Rutas Cuantitativa, Cualitativa y Mixta. Editorial: Mc Graw - Hill Interamericana S.A. México. 714 p.

Ley $N^{\mathrm{a}} 28015$ Ley de Promoción y Formalización de la Micro y Pequeña Empresa en el Perú. 2003. https://www.ilo.org/dyn/ natlex/natlex4.detail? $p \_$isn $=65715 \& \mathrm{p}$ lang $=\mathrm{es}$

Millones, R.; Barreno, E.; Vásquez, F.; Castillo, C. 2016. Libro: Estadística Aplicada a la Ingeniería y los Negocios. Editorial: Universidad de Lima. Fondo Editorial. Perú. $500 \mathrm{p}$.

Ortega, F. 2020. Libro: Resultado, Rentabilidad y Caja. Editorial: ESIC. España. 300p.
Parraga Franco, S.; Pinargote Vásquez, N.; García Álava, C. y Zamora Sornoza, J. 2021. Indicadores de gestión financiera en pequeñas y medianas empresas en Iberoamérica: una revisión sistemática. Dilemas contemp. educ. Política valores. México. Vol.8 (2). 1-24. http://www.scielo.org.mx/scielo. php script $=$ sci_arttext\&pid $=$ S 2007 78902021000400026\&lang=es

Rodríguez, P. 2016. Gestión Financiera en PyMES. Revista Publicando. Vol.3 (8).

https://dialnet.unirioja.es/servlet/ articulo? codigo $=5833410$

Sanna, A. 2018. Libro: El Cuadro de Mando Integral. Mejore su reflexión Estratégica. Editorial: Titivillus. México. 21 p.

Sánchez, H. 2020. Libro: Análisis FOSA o DAFO. Editorial: Bubok Publishing S.L. Madrid. España. 206 p.

Sociedad de Comercio Exterior del Perú COMEX. 2020. Artículo: Las MYPE peruanas y su realidad ante la crisis. Disponible en: https://www.comexperu.org.pe/articulo/lasmype-peruanas-en-2019-y-su-realidad-antela-crisis. Perú. 4 p.

Superintendencia de Aduanas y Administración Tributaria [SUNAT]. 2019. Decreto Supremo $N^{\circ}$ 298-2018-EF. Disponible en: https://www.sunat.gob.pe/ indicestasas/uit.html

Tanta, S. 2020. La contabilidad gerencial y su influencia en la gestión administrativa de las micro y pequeñas empresas de telefonía móvil en la ciudad de Ayacucho, 2019. Tesis de Maestría en Contabilidad con mención en finanzas. Universidad Católica los Ángeles Chimbote.

Disponible en http://repositorio.uladech.edu. pe/handle/123456789/18504? show=full

Wasbaldo, J. 2018. La Contabilidad Gerencial y la toma de decisiones administrativas en el Instituto de Manejo y Medio Ambiente 2018. Tesis de Maestro en Gestión Pública. Universidad Cesar Vallejo. Disponible en https://repositorio.ucv.edu.pe/ handle/20.500.12692/33926? show=full 